\title{
Una visión holística de la depresión en adultos mayores colombianos
} A Holistic View of Depression in Colombian Older People

Recibido: 29 Septiembre 2020 | Aceptado: 20 Noviembre 2020

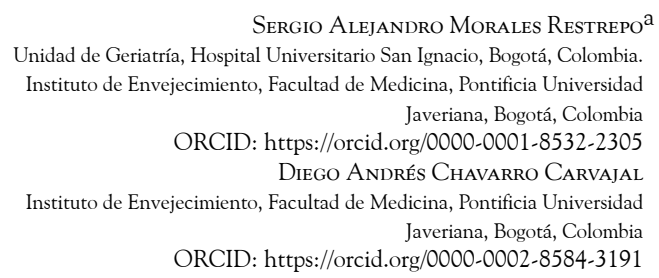

\section{RESUMEN}

La población mundial está cambiando en la esfera sociodemográfica, con un aumento importante de la proporción de ancianos, sobre todo en Colombia. Por esto, es esencial el estudio de las enfermedades que afectan a esta población, las enfermedades crónicas que han aportado a la mortalidad en los últimos años y las patologías mentales, especialmente por el aumento de su incidencia y prevalencia. De estas, los trastornos del afecto son los de mayor prevalencia en ancianos, pues cobran gran importancia el estudio de sus factores de riesgo, métodos diagnósticos y alternativas de tratamiento. Al respecto, se plantean factores biológicos, sociales, culturales y del entorno que modifican la incidencia y presentación clínica de estos trastornos, especialmente de la depresión. Se han publicado estudios sobre violencia y adversidades en la infancia asociado al riesgo de presentar depresión en los ancianos. El objetivo del artículo es describir el posible impacto del entorno y ambiente social colombiano en la depresión en ancianos.

Palabras clave

depresión; experiencias adversas de la infancia; anciano; violencia; medio social.

a Autor de correspondencia: morales.sergio@javeriana.edu.co

Cómo citar: Morales Restrepo SA, Chavarro Carvajal DA. Una visión holística de la depresión en adultos mayores colombianos. Univ. Med. 2021;62(1). https:/ /doi.org/10.11144/Javeriana.umed62-1.holi

\section{ABSTRACT}

The world population is changing at the sociodemographic level, there has been a progressive increase in the elderly population globally, particularly in Colombia. It is essential to study those diseases that afflict this population, the chronic diseases that have been among the leading causes of death in this age group, and mental illness, especially due to the progressive increase in their prevalence and incidence. Affective disorders are the most prevalent mental pathology in the elderly, so clinical manifestations, diagnostic methods, and treatment alternatives should be studied. There has been described multiple risk factors that have modified its incidence and clinical presentation. For several years, studies on childhood experiences, including violence and adversity and the possible association with depression in the elderly, have been published. This article aims to describe the importance of the Colombian social context as a possible risk factor for developing depression in older people and their consequences

Keywords

depression; adverse childhood experiences; aged; violence; social environment. 


\section{Introducción}

La transición demográfica es un proceso que se ha estado desarrollando desde hace varios años en el mundo, el cual ha generado importantes cambios demográficos que se evidencian en la inversión de la pirámide poblacional, acompañada del aumento de la proporción de ancianos dentro de los países. Colombia no ha sido ajena a este proceso, lo cual se evidenció en los resultados del último censo poblacional $(1,2)$. Ante el aumento de la población de este grupo etario, surge la prioridad de evaluar las condiciones de salud, bienestar y sociodemográficas.

Sumado a los cambios demográficos ya descritos, los cambios sociales y en salud pública han modificado la prevalencia de las enfermedades en los ancianos. Dentro de estos, se ha visto una disminución progresiva de la incidencia de enfermedades infecciosas y un aumento de la prevalencia de enfermedades crónicas no transmisibles (3). Por otro lado, entre más años tenga un anciano, hay una mayor prevalencia e incidencia de patología mental (4). De este último grupo, se debe hacer hincapié en el trastorno neurocognitivo de diferentes etiologías, y no menos importante, los trastornos del afecto como la depresión y la ansiedad (5).

La presente revisión temática tiene como objetivo abarcar la depresión en la población anciana desde una visión global, que permita conocer las múltiples dimensiones que en ellas interviene, con el fin realizar un mejor abordaje a esta patología con prevalencia en aumento.

\section{Contenido: envejecimiento poblacional}

El envejecimiento poblacional es un fenómeno que acontece desde hace varios años. Se puede ver como un logro de los avances de la salud pública y de los sistemas de salud; sin embargo, este y el aumento progresivo de la esperanza de vida plantean retos a los servicios de salud y a la sociedad en general. Por un lado, porque la legislación de algunos países no se ha ajustado a esta realidad y porque tampoco se han invertido suficientes recursos para lograr la infraestructura que ofrezca una atención integral a esta población. Por otro, empieza a tomar gran importancia la salud y el bienestar de los ancianos, por lo que es preciso que se haga hincapié en la prevención primaria, secundaria y terciaria de las enfermedades que a esta población afecta y el impacto que generan en su calidad de vida y grado de independencia (6).

En el 2015 se llevó a cabo en nuestro país la encuesta Salud, Bienestar y Envejecimiento (SABE), la cual se aplicó mediante cuestionarios a una población representativa de Colombia de 65 años o más e incluyó datos de 232.694 personas en hogares de zonas urbanas y rurales. La mediana de edad fue de 69 años, se contaron 5,5 años de escolaridad en promedio y se evaluaron las limitaciones para el acceso a empleo, a pensiones y a servicios de salud. Además, la prevalencia de hipertensión fue del 60 $\%$; de enfermedad isquémica cardiaca, del $14 \%$, y de enfermedad pulmonar crónica, del 11,4\%, como las enfermedades crónicas no transmisibles más prevalentes. Respecto a la patología mental, el $41 \%$ de los encuestados reportó síntomas depresivos, con mayor prevalencia en estratos socioeconómicos altos y menor prevalencia a mayor edad. Más allá de la alta prevalencia de las diferentes patologías, vale la pena poner el relieve en las herramientas diagnósticas y los tratamientos disponibles para cada una de ellas. Por citar un ejemplo, la enfermedad isquémica cardiaca tiene una presentación clínica específica que permite al clínico un diagnóstico temprano y realizar un tratamiento adecuado (7). Por otra parte, cuando hablamos de patología mental, debemos saber que el cuadro clínico surge de forma heterogénea y ello hace que su diagnóstico requiera una evaluación longitudinal de días, semanas, meses o incluso años. Por otro lado, se desconocen los mecanismos fisiopatológicos de algunas enfermedades mentales, motivo por el cual, como en el caso de la depresión, el $33 \%$ responderá al manejo inicial, el $33 \%$ precisará cambió de la medicación y el $34 \%$ restante no responderá al manejo farmacológico $(8,9,10$, 11). 


\section{Depresión}

\section{Definición y epidemiología}

La depresión en el anciano, según lo definido en la quinta edición del Manual diagnóstico y estadístico de los trastornos mentales (DMS-V) y la décima edición de la Clasificación Internacional de Enfermedades (CIE-10), es aquella que se presenta en personas mayores de 65 años de edad, con una prevalencia estimada del $1 \%$ hasta el 12,3\%, de acuerdo con datos de la Encuesta SABE Colombia (3). En esta se encontró, en cuanto al género masculino, que el $16,4 \%$ tenía depresión leve y el 5,7 \% ya tenía depresión establecida; en las mujeres, el 18,7\% tenía depresión leve, y el 10,1\%, depresión ya establecida. En cuanto al aumento de edad y prevalencia de esta, entre los 60 y los 69 años, el 23,8 \% tenía depresión; entre los 70 y los 79 años, el 25,8\%, y para los mayores de 80 años, una prevalencia del $34,5 \%$, con un aumento significativo (12).

En el mundo se calcula una prevalencia del 1 $\%$ al $4 \%$ de depresión mayor en ancianos, con una incidencia del 0,15\% al año; además, se ha encontrado el doble de afectación en las mujeres respecto a los hombres y un aumento progresivo de la prevalencia e incidencia al doble después de los 85 años. Sin embargo, cabe mencionar que la prevalencia de depresión menor puede llegar hasta un $13 \%$ en esta población (13). Según los estudios, esta afecta con mayor frecuencia a aquellos ancianos con enfermedades crónicas, deterioro cognitivo o discapacidad $(14,15)$. También es más frecuente encontrar depresión en ancianos que se encuentran hospitalizados o al cuidado de hogares geriátricos. Predicciones de la Organización Mundial de la Salud en el 2015 indicaban que para el 2020, la depresión sería la tercera causa de discapacidad mundial $(16,17$, 18).

Podemos encontrar también la presencia de síntomas depresivos que no cumplen los criterios para el diagnóstico depresión, ya sea por los criterios CIE-10 o del DMS-V, los cuales se documentan dos o tres veces más que la enfermedad en sí. A pesar de lo anterior, estos síntomas afectan clínicamente a los ancianos y llevan a una reducción de la calidad de vida, disminución en el funcionamiento social, un mayor riesgo de presentar depresión a posteriori, entre otras consecuencias $(16,19)$. Al respecto, es importante aclarar que la causa de algunas patologías mentales, especialmente de los trastornos del afecto, es multifactorial, sin que haya podido establecerse actualmente una sola etiología de la depresión, y en la cual hasta el momento se considera que intervienen múltiples factores, como los genéticos, los sociales, los biológicos, los socioambientales y los psicológicos, que influyen de forma diferencial en cada etapa de la vida del individuo (13).

\section{Impacto y consecuencias en el anciano}

Una de las grandes dificultades al abordar la depresión en el anciano, no es el tratamiento en sí, sino el diagnóstico y la dificultad para llegar a él. En general, el diagnóstico de la depresión requiere un seguimiento longitudinal para lograr una adecuada caracterización de los síntomas; sin embargo, muchas veces solo es posible hacer diagnósticos retrospectivos cuando ya se encuentra en un estadio avanzado de la enfermedad. La depresión repercute en muchos aspectos tanto a los ancianos como a sus cuidadores. Vemos cómo la depresión se asocia con demencia, discapacidad o enfermedades crónicas - por ejemplo, enfermedad cardiovascular, inmovilismo, entre otras- $(20,21)$. Así, sus consecuencias no solo están en la órbita de la salud, sino también en la calidad de vida tanto para los pacientes como para sus familias (22).

El suicidio es en sí un gran reto y, por lo tanto, requiere un abordaje diferencial en los ancianos, dados los registros sobre infradiagnóstico del $32 \%$ al $50 \%$, acompañado de tratamiento subóptimo hasta en la mitad de los casos (14). Aunque los intentos de suicidio han sido históricamente más frecuentes en personas jóvenes, datos recientes muestran que la mayor tasa de suicido se encuentra en adultos mayores 
en casi todos los países, al punto que ha alcanzado 48,7/100.000 en hombres blancos en Estados Unidos y 140/100.000 en hombres que viven en China. Por otra parte, de forma llamativa, datos obtenidos de Estados Unidos evidencian que la relación de intentos de suicidio y suicidio consumado en jóvenes es de 36:1; mientras que en los ancianos es de 4:1, lo cual indica que la letalidad de los intentos es alta, especialmente en aquellos mayores de 70 años (23).

Dentro de las características del suicidio en este grupo etario se puede encontrar que se suicidan con menos intentos previos y suelen visitar al médico antes de llevarlo a cabo, como una forma de mostrar incomodidad con su vida $(14,24,25)$. Dado lo anterior, es importante que el personal de salud que atiende a esta población, especialmente en el ámbito de atención primaria, tengan en cuenta las diferentes formas en las que los ancianos expresan de forma indirecta la intención de realizar un acto de autolesión. Dentro de estas pistas, encontramos expresiones como "soy una carga para mi familia" o "ellos serían más felices si yo no estuviera vivo/a" (26).

\section{Factores de riesgo}

Los factores de riesgo menos estudiados, pero igualmente importantes, son las situaciones y eventos sufridos a través de la vida, de los cuales haremos hincapié en los eventos adversos ocurridos en la infancia. Estos tomaron gran importancia, debido a la alta prevalencia de enfermedad mental en sobrevivientes de tragedias mundiales, como lo fue la Segunda Guerra Mundial y el Holocausto. Un estudio del año 2000 comparó una muestra poblacional de 74 años entre sobrevivientes del Holocausto y aquellos de la misma edad, pero que no sufrieron estos hechos históricos, en la cual se incluyó un total 194 personas. Se encontró que aquellos ancianos expuestos a la violencia eran más vulnerables, con mayor riesgo de desarrollar patología mental, como depresión y estrés postraumático. Se describieron múltiples determinantes para desarrollar respuesta al trauma de forma inmediata o en años posteriores y se postularon tres factores principales: el evento traumático en sí; factores de personalidad, como el locus de control, definido como la atribución que una persona lleva a cabo sobre si el esfuerzo que realiza es o no contingente a su conducta, y factores del entorno de cada individuo, como lo son el apoyo social y familiar $(27,28,29)$.

Teniendo en cuenta estos acontecimientos presentados en aquellos veteranos de guerra, se empezó a plantear una hipótesis sobre la relación de eventos en la infancia y desenlaces presentados en la adolescencia y durante la vejez, especialmente después de los 65 años (30). En 1998, Felliti et al. (citados en [31]) publicaron un documento sobre las situaciones adversas en la infancia y su relación con desenlaces mentales y físicos. Desde entonces se empezaron a publicar estudios en algunos países que intentan reforzar esta hipótesis.

En 2019, el Centers for Disease Control and Prevention emitió un documento al respecto, en el que hacía hincapié en la mejor evidencia disponible sobre esta temática. Definió las situaciones adversas en la infancia como los eventos potencialmente traumáticos que ocurren desde el nacimiento hasta la adolescencia, es decir, desde los 0 a los 17 años. En este grupo incluyen violencia, abuso o negligencia; ser testigo de violencia familiar, y tener un familiar que ha tenido un intento de suicidio o ha consumado uno $(32,33)$. Estos eventos pueden ser emocionalmente difíciles y estresantes, y potencialmente generar efectos a corto y largo plazo. El impacto del evento estresor depende de la naturaleza, la frecuencia y gravedad del suceso, la historia previa de trauma o la falta de apoyo que ayude a superar estos eventos. Por otro lado, las consecuencias no son netamente físicas y mentales, pues el haber sufrido un evento negativo en la infancia puede repercutir en la educación, el empleo, el aprendizaje y la dificultad en el desarrollo escolar, laboral y social, lo cual influye en costos para las familias, las comunidades y la sociedad en general (34). Actualmente, existe evidencia fuerte que asocia estos estresores con el desarrollo de trastornos del afecto, como es el caso de la depresión (35). 
Esto refuerza que el desarrollo de la personalidad y de la vida de las personas depende de un modelo que incluye dos componentes: uno, los factores de riesgo que presenta, y otro, las oportunidades para hacer contrapeso a las primeras.

Basándose en lo anterior, se empezaron a realizar estudios sobre los eventos positivos en la infancia; sin embargo, no ha sido posible estandarizar su medición y establecer su prevalencia. A pesar de ello, se han publicado estudios con muestras no representativas, los cuales han explorado la interacción entre las situaciones adversas y las positivas en la infancia (cercanía con familiares y amigos, apoyo social, lealtad, amor y acompañamiento). Estas se asociaron con menores tasas de embarazos en la adolescencia, menor patologías por abuso de sustancias y menor tasa de enfermedad mental en personas jóvenes. Se plantea, entonces, que estas situaciones positivas podrían intervenir para prevenir o disminuir las consecuencias de situaciones adversas que se presentan en la infancia o a través del curso de vida $(21,35)$.

\section{Contexto colombiano}

$\mathrm{Al}$ avanzar en los hechos históricos de guerras globales, en la misma época, cuando varios países luchaban en la Segunda Guerra Mundial, en Latinoamérica empezaban a surgir varios movimientos de personas alzadas en armas, las cuales defendían diferentes ideologías, pero que estaban enmarcadas en la ilegalidad, el narcotráfico y la violencia. Respecto a la violencia, la Organización Mundial de la Salud la clasificó en 1) violencia colectiva, aquella provocada por los Estados o grupos políticos; 2) violencia interpersonal, la que se produce en un ámbito intrafamiliar o de pareja, y 3) en violencia autoinfligida, que representa el comportamiento suicida y las autolesiones $(36,37,38)$ (tabla 1). En Colombia se han vivido históricamente estos tres tipos de violencia. Al respecto, vale recordar que durante los últimos cincuenta años, en nuestro país, se han identificado dos periodos críticos de violencia: el primero durante la década de 1980, que perduró varias décadas y cobró la vida de muchas personas y, el segundo, a principios de la década de 1990, con grandes cambios políticos y sociales, como fue el caso de la Asamblea Nacional Constituyente de 1991 (36, $39,40)$.

\section{Tabla 1}

Clasificación de la violencia según el autor y la naturaleza

\begin{tabular}{|l|l|l|}
\hline \multicolumn{1}{|c|}{ Tipos } & \multicolumn{1}{|c|}{ Subtipos } & \multicolumn{1}{c|}{ Formas de violencia } \\
\hline $\begin{array}{l}\text { Violencia } \\
\text { colectiva }\end{array}$ & $\begin{array}{l}\text { Estados, grupos políticos, } \\
\text { milicias u organizaciones } \\
\text { terroristas }\end{array}$ & $\begin{array}{l}\text { Social (crimen organizado, pandillas); } \\
\text { política (conflictos armados, terrorismo); } \\
\text { económica (alteración actividad económica) }\end{array}$ \\
\hline $\begin{array}{l}\text { Violencia } \\
\text { interpersonal }\end{array}$ & $\begin{array}{l}\text { Intrafamiliar o de pareja; } \\
\text { comunitaria }\end{array}$ & $\begin{array}{l}\text { Maltrato o violencia entre grupo familiar; } \\
\text { pandillas, agresiones sexuales, violencia en } \\
\text { ámbitos laborales y educativos }\end{array}$ \\
\hline Autoinfligida & $\begin{array}{l}\text { Comportamiento suicida y } \\
\text { autolesiones }\end{array}$ & $\begin{array}{l}\text { Ideas, planeación y consumación; maltrato y } \\
\text { mutilaciones }\end{array}$ \\
\hline
\end{tabular}

Fuente: elaboración propia con información tomada de $(36,37,55)$.

De estos datos históricos no solo queda una cifra de personas asesinadas, niños huérfanos, mujeres viudas y familias destrozadas, se empieza ver — consecuente con lo anteriorun incremento en el desplazamiento forzado, especialmente del área rural hacia los centros urbanos, lo que conlleva un cambio repentino en el curso de vida y un impacto importante en los ancianos, con cifras aproximadas del $8,5 \%$ de afectación en este grupo etario, el cual empezó a hacer parte de las cifras de desempleo y desnutrición, como ha sucedido históricamente en países de Latinoamérica, por ejemplo, México (5). Datos del estudio SABE Colombia mostraron que el $15,4 \%$ de esta población sufrió desplazamiento alguna vez en la vida por violencia o conflicto armado, con mayor prevalencia en regiones como la Orinoquía/ Amazonía, con un 26,6\%, o la región atlántica, con el $21 \%$ (40). Al igual que lo sucedido con los veteranos del Holocausto, probablemente en Colombia haya ancianos con enfermedad mental que podrían tener alguna asociación con los sucesos vividos durante su infancia y adolescencia, en un país en el cual durante la época primaba la pobreza y las pésimas condiciones de salud e higiene y una mínima cobertura de servicios públicos $(36,41)$.

Quedó desde entonces un país con un importante índice de desempleo, de pobreza, de inequidad económica y social. De forma paralela 
a la violencia política y social, se vivía una guerra dentro de las familias y pequeñas comunidades, especialmente en aquellas con algún grado de vulnerabilidad, lo que se conoce como maltrato (42). Aunque hacer una medición del maltrato a personas mayores presenta diversas dificultades por los instrumentos de medición y tipos de estudio, se estima que su prevalencia en la comunidad de Norteamérica y Suramérica oscila entre el 2,2 \% y el 44,6\% (43). Estas condiciones adversas, violencias y maltrato fueron más evidentes en determinados grupos poblaciones, como se encontró en un análisis secundario del estudio SABE Colombia, en el cual se documentó cómo las mujeres mayores tuvieron menos oportunidades académicas, económicas y sociales durante su juventud, y ello llevó así a que haya mayor prevalencia de mujeres analfabetas, menor acceso a pensión, mayor índice de pobreza y mayor exposición a hechos de violencia respecto a los hombres ancianos colombianos $(9,44)$.

Así es como el desarrollo de la mayoría de los países ha sido ampliamente influenciado por su historia y las consecuencias de esta. Surgieron entonces varios grupos armados al margen de la ley, uno de los cuales se desmovilizó mediante un proceso liderado por el expresidente Juan Manuel Santos Calderón. Posterior a esto, disminuyeron notablemente los actos de violencia a la población civil, y salieron a la luz pública otros problemas sociales, como la pobreza, el hambre, el desempleo y la violencia intrafamiliar, sobre los cuales se trabaja actualmente desde la política pública y organizaciones no gubernamentales (45).

Entender la historia reciente de nuestro país es importante, porque nos permite saber de dónde surgen las dificultades que se viven actualmente, como son la pobreza y la corrupción, y porque nos predice, aunque de forma no exacta, las consecuencias que se podrían presentar en unos años si no tomamos medidas a tiempo. Conocer nuestra historia nos permite hacer todo lo posible para no repetirla $(39,46)$.

De esta forma, durante el curso de vida, algunos ancianos han sufrido diferentes dificultades, del ámbito social y económico; otras debido al contexto de violencia del país $\mathrm{y}$, por supuesto, algunas condiciones generadas por las afectaciones de su salud, como son las enfermedades crónicas y aquellas que generan un gran impacto, como el cáncer.

A pesar de no poder generar una causalidad entre estos acontecimientos y la presencia de síntomas depresivos en los ancianos, principalmente por la limitación de los estudios transversales y la imposibilidad de analizar una cohorte con más de 50 años de seguimiento, es relevante conocer todo aquello que repercute en esta población, y a raíz de este conocimiento, implementar medidas y herramientas que permitan subsanar algunas dificultades, y en caso de no ser posible, llevar a cabo una intervención integral que minimice las consecuencias fatales, como es el caso del suicidio.

\section{Discusión}

La población anciana ha aumentado tanto en América Latina como en Colombia (1). Los Estados deben priorizar la inversión en ella, poniendo el acento en su bienestar físico, social y mental, los cuales guardan una estrecha relación. $\mathrm{Al}$ respecto se deben crear protocolos con el fin de brindar apoyo social y económico a los ancianos; además, diagnosticar su situación de salud, con el fin de enfocar las medidas preventivas de las patologías más prevalentes. Esta intervención se debe centrar en evitar las posibles consecuencias ya descritas en otros estudios, como lo es la discapacidad, las enfermedades crónicas como la cardiovascular y, no menos importante, el suicidio $(24,47)$.

Para lograr lo anterior, es fundamental una valoración completa e integral a los ancianos, con el fin de proporcionarles un tratamiento dirigido, teniendo siempre como pilar el contexto y el curso de vida de cada uno de ellos (48). Se debe atender a la población anciana con un enfoque diferencial e integral, en la cual interviene un equipo multidisciplinario, centrado en la valoración geriátrica integral por parte de geriatras, la cual incluye todas sus dimensiones. 
Al respecto, la cantidad de personal especializado en esta atención es insuficiente para cubrir a toda la población anciana mundial o de nuestro país $(49,50)$. Aquí es donde cobra gran importancia la atención primaria en salud, que abarca gran parte del territorio nacional, con la finalidad de unificar los protocolos y las medidas necesarias para lograr de forma efectiva y oportuna esta atención (51). Para lo anterior, se propone crear grupos de atención médica encabezados por especialistas y conformados por un gran número de profesionales en salud, entre ellos médicos generales, médicos familiares, psicólogos y terapeutas, con el fin de realizar un adecuado diagnóstico, tratamiento y rehabilitación. Todo ello para lograr llegar a todas las áreas rurales y urbanas donde residan los beneficiados. Debemos defender a toda costa la calidad de vida de los ancianos y trabajar desde los diferentes frentes para lograr esta meta.

\section{Conclusiones}

La transición demográfica es un proceso irreversible que aumenta de forma significativa la proporción de ancianos en el mundo. Ello impone retos como la inversión en el área social y en la prestación de servicios de salud de calidad. En este último, se debe hacer hincapié en el bienestar físico y mental, mediante la prevención de enfermedades por medio de herramientas como la actividad física y la alimentación adecuada, con el fin de promover un envejecimiento exitoso (52). Por otro lado, hacer un esfuerzo grande en el diagnóstico de las enfermedades que prevalecen en este grupo etario.

El bienestar debe incluir también la salud mental, dentro de la cual prevalecen notoriamente los trastornos del afecto, como la depresión y la ansiedad. Se deberán reforzar las herramientas diagnósticas y de tratamiento para la enfermedad mental, pero sobre todo valorar los factores de riesgo modificables para estas patologías, como lo son los factores sociales, los económicos y los demográficos de cada uno de los ancianos de un país (53). Este último aspecto es vital evaluarlo en los adultos mayores colombianos, teniendo en cuenta todos los hechos históricos que han experimentado en el transcurso de sus vidas, como los ya mencionados.

Consideramos que si bien se incluyeron en el análisis estudios poblacionales como la Encuesta SABE, reconocemos que los estudios transversales son una única mirada de la realidad de las personas, por lo cual pueden existir otros factores o variables que repercutan en los resultados encontrados y que se deban tener en cuenta para posteriores análisis, a fin de poder determinar causalidad entre los factores, pero planteando posibles hipótesis y generando así conciencia en todos aquellos profesionales de la salud que hacen parte de la atención integral de esta población. Cabe resaltar que la depresión y los trastornos neurocognitivos son los trastornos neuropsiquiátricos más prevalentes en el adulto mayor, en los cuales intervienen múltiples factores, pero en los cuales la incidencia y prevalencia varía de acuerdo con el desarrollo socioeconómico de los países. Estas patologías generan un gran impacto en los ancianos, sus familias y en el país en general, que, como Estado, son los garantes de promover su bienestar y garantizar su calidad de vida en el transcurso de los años.

Por último, cabe resaltar que durante el curso de vida no solo se pueden presentar situaciones adversas, sino que también se viven situaciones que se consideran como factores positivos y que modifican la balanza, pues evitan en muchos casos las consecuencias de estos hechos desfavorables durante los primeros años de vida (54). Esto es de vital importancia.

\section{Fuente de financiación}

No se contó con fuente de financiación.

\section{Conflicto de intereses}

Los autores declaran que no existe ningún conflicto de intereses. 


\section{Referencias}

1. Floréz CE, Villar L, Puerta $\mathrm{N}$, Berrocal LF. El proceso de envejecimiento de la población en Colombia. Bogotá: Fundación Saldarriaga Concha; 2015.

2. Flórez JE. ¿Transición demográfica en Colombia? Cuad Geogr Rev Colomb Geogr. 1995;5(2):133-46.

3. Cano Gutiérrez C, Germán Borda M, Samper-Ternent R, ChavarroCarvajal DA, Escudero de Santacruz C. Iluminando las decisiones e intervenciones públicas para la población adulta mayor: Estudio Sabe Bogotá. Medicina (B Aires). 2018;40(2):191-207.

4. Bastidas Bilbao H. Enfermedades médicas y depresión en el adulto mayor: características comunes y relación etiológica. Rev Psicol. 2014;32(2):191-218.

5. Pando Moreno M, Aranda Beltrán C, Alfaro Alfaro N, Mendoza Roaf P. Prevalencia de depresión en adultos mayores en población urbana. Rev Esp Geriatr Gerontol. 2001 Jan;36(3):140-4. http://dx.doi.org/10.1 016/S0211-139X(01)74704-X

6. Jadad A, Cabrera A, Lyons R, Martos F, Smith R, editores. Prevention and health promotion. En When people live with multiple chronic diseases: a collaborative approach to an emerging global challenge. Granada: Escuela Andaluza Salud Pública; 2010. p. 59-88.

7. Guerrero A, Trongue J. Enfermedad coronaria crónica: aspectos clínicos, epidemiológicos y evolutivos. Prosac. 2008;3(1):1-18.

8. González Quiñones JC. Resumen: Sabe Colombia 2015: Estudio Nacional de Salud, Bienestar y Envejecimiento. Cart Comunitaria. 2017;25(144):24.
9. Santacruz M, Residente E, Esteban H, Rosero P. Comorbilidades asociadas a depresión en viejos según la Encuesta Sabe Bogotá. 2017;5.

10. Domínguez LJ, Galioto A, Ferlisi A, Pineo A, Putignano E, Belvedere M, et al. Ageing, lifestyle modifications, and cardiovascular disease in developing countries. J Nutr Health Aging. 2006;10(2):143-9.

11. Castro Lizárraga M, Ramírez Zamora S, Aguilar-Morales LV, Díaz de Anda MV. Factores de riesgo asociados a la depresión del adulto mayor. Neurol Neurocir Psiquiatr [internet]. 2006;39(4):132-7. Disponible en: https ://www.medigraphic.com/pdfs/revneun eupsi/nnp-2006/nnp064b.pdf

12. Gómez-Restrepo C, Bohórquez A, Pinto Masis D, Gil Laverde JFA, Sepúlveda MR, Díaz-Granados N. Prevalencia de depresión y factores asociados con ella en la población colombiana. Rev Panam Salud Publica [internet]. 2004;16(6):378-86. Disponible en: https://iris.paho.org/bits tream/handle/10665.2/8150/23682.pdf ?sequence $=1$ \&isAllowed $=\mathrm{y}$

13. Alexopoulos GS. Elderly depression. Lancet. 2005;365(9475):1961-70. https://doi.o rg/10.1016/S0140-6736(05)66665-2

14. Urbina Torija JR, Flores Mayor JM, García Salazar MP, Torres Buisán L, Torrubias Fernández RM. Síntomas depresivos en personas mayores: prevalencia y factores asociados. Gac Sanit. 2007;21(1):37-42. http://dx.doi. org/10.1157/13099119

15. Estrada Restrepo A, Cardona Arango D, Segura Cardona AM, Ordóñez Molina J, Osorio Gómez JJ, Chavarriaga Maya LM. Depressive symptoms in institutionalized elderly people and factors associated. Univ Psychol. 2013;12(1):81-94. 
16. Borza T, Engedal K, Bergh S, Selbek G. Older people with depression. Tidsskr Nor Laegeforen. 2019 Nov 4;139(16). Norwegian, English. https:/ /doi.org/10.4045/tidsskr.18.0968

17. Cooper R. Diagnostic and statistical manual of mental disorders (DSM). Vol. 44, Knowledge Organization; 2017. p. 668-76.

18. American Psychiatric Association. Diagnostic and statistical manual of mental disorders. 5. $\mathrm{a}$ ed. Washington; 2013.

19. Gebretsadik M, Jayaprabhu S, Grossberg GT. Mood disorders in the elderly. Med Clin North Am. 2006;90(5):789-805.

20. Martínez Hernández O. Depresión en el adulto mayor. Rev Medica Electron. 2007;29(5):514-25.

21. Cordeiro RC, Santos RCD, Araújo GKN, Nascimento NM, Souto RQ, Ceballos AGDC, et al. Mental health profile of the elderly community: a cross-sectional study. Rev Bras Enferm. 2020;73(1):e20180191. https://doi.org /10.1590/0034-7167-2018-0191

22. Sivertsen H, Bjørkløf GH, Engedal K, Selbæk G, Helvik AS. Depression and quality of life in older persons: A review. Dement Geriatr Cogn Disord. 2015 Oct 14;40(5-6):311-39.

23. De Souza Minayo MC, Cavalcante FG. Suicide in elderly people: A literature review. Rev Saude Publica. 2010;44(4):750-7.

24. Minayo MCS, Sousa JRA, Cavalcante FG, Mangas RMN. Autópsias psicológicas sobre suicídio de idosos no Rio de Janeiro. Cienc e Saude Coletiva [internet]. 2012;17(10):2773-81. Disponible en: http://www.cienciaesaudecoletiva. com.br/artigos/autopsias-psicologicas-s obre-suicidio-de-idosos-no-rio-de-jane iro/7855? id $=7855$
25. Barrero SAP. Factores de riesgo suicida en el anciano. Cienc e Saude Coletiva. 2012;17 (8):2011-6.

26. Sorrell JM. Suicide in older adults. J Psychosoc Nurs Ment Health Serv. 2020;58(1):17-20.

27. Landau R, Litwin H. The effects of extreme early stress in very old age. J Trauma Stress. 2000;13(3):473-87.

28. Joffe C, Brodaty $\mathrm{H}$, Luscombe G, Ehrlich F. The Sydney Holocaust study: posttraumatic stress disorder and other psychosocial morbidity in an aged community sample. J Trauma Stress. 2003;16(1):39-47.

29. O'Rourke N, Carmel S, Bachner YG. Does early life trauma affect how depression is experienced by Holocaust survivors in late life? Aging Ment Heal [Internet]. 2018 May 4 [citado 2020 feb 14];22(5):662-8. Disponible en: https://www.tandfonline.com/doi/f ull/10.1080/13607863.2017.1286458

30. Scoglio AAJ, Shirk SD, Mazure C, Park CL, Molnar BE, Hoff RA, et al. It all adds up: addressing the roles of cumulative traumatic experiences on military veterans. Child Abus Negl [internet]. 2019 Dec [citado 2020 feb 14];98:104227. Disponible en: https://inkinghub.elsevier.com/ret rieve/pii/S0145213419304041

31. Frampton NMA, Poole JC, Dobson KS, Pusch D. The effects of adult depression on the recollection of adverse childhood experiences. Child Abus Negl. 2018 Dec 1;86:45-54. https://doi.org/10.1016/j.c hiabu.2018.09.006

32. Kremer P, Ulibarri M, Ferraiolo N, Pinedo M, Vargas-Ojeda AC, Burgos $\mathrm{JL}$, et al. Association of adverse childhood experiences with depression in latino migrants residing in Tijuana, Mexico. Perm J [internet]. 2018 [citado 2020 ene 21];23:18-031. Disponible 
en: https://www.ncbi.nlm.nih.gov/pub $\mathrm{med} / 30589403$

33. Henry BF. Typologies of adversity in childhood \& adulthood as determinants of mental health \& substance use disorders of adults incarcerated in US prisons. Child Abus Negl. 2020 Jan 1;99.

34. Chen H, Lu MC, Ayers JF, Wiesman J. Preventing adverse childhood experiences. Centers for Disease Control and Prevention [internet]. 2019. Disponible en: https://www.cdc.gov/violencepreve ntion/childabuseandneglect/aces/fastfa ct.html

35. Bethell C, Jones J, Gombojav $\mathrm{N}$, Linkenbach J, Sege R. Positive childhood experiences and adult mental and relational health in a statewide sample: associations across adverse childhood experiences levels. JAMA Pediatr [internet]. 2019 Nov 4 [citado 2020 feb 14];173(11):1-10. Disponible en: https://jamanetwork.com/journals/j amapediatrics/fullarticle/2749336

36. Cano C, De Santacruz C, Ternent RS, Borda M, Medina M, Chavarro D, et al. Las personas viejas en colombia memoria viva de las violencias y de las resistencias. Rev Asoc Colomb Gerontol Geriatr [internet]. 2016;30(1):2206-57. Disponible en: ht tp://acgg.org.co/pdf/pdf_revista_16/art iculo2revista1-2016.pdf

37. Arce y Temes Á. Los conflictos en Latinoamérica. Nação e Def; 1984. p. 26-46.

38. Cano SM, Cardona D. Factores asociados al maltrato del adulto mayor de Antioquia, 2012. Rev Fac Nac Salud Pública. 2015;33(1):67-74.

39. Bello Montes C. La violencia en Colombia: análisis histórico del homicidio en la segunda mitad del siglo XX. Criminalidad. 2008;50(1):73-84.

40. Curcio C-L, Vanegas JH, Palacio MC, Corchuelo Ojeda J. Vejez y desplazamiento forzado en Colombia. Colomb Med. 2019;50(2):52-66.

41. Barel E, Van IJzendoorn MH, SagiSchwartz A, Bakermans-Kranenburg MJ. Surviving the holocaust: A metaanalysis of the long-term sequelae of a genocide. Psychol Bull. 2010 Sep;136(5):677-98.

42. Organización Mundial de la Salud. Informe mundial sobre violencia y salud [internet]. Vol. 1. Washington; 2003. Disponible en: http://iris.paho.or $\mathrm{g} / \mathrm{xmlui} /$ bitstream/handle/123456789/ 725/9275315884.pdf? sequence $=1$ \&is Allowed $=\mathrm{y}$

43. Curcio C-L, Payán-Villamizar C, Jiménez A, Gómez F. Maltrato en adultos mayores colombianos y su asociación con condiciones socioeconómicas y funcionalidad. Colomb Med. 2019;50(2):77-88. https ://doi.org/10.25100/cm.v50i2.4013

44. Santacruz C de, Chavarro Carvajal DA, Venegas-Sanabria LC, Gama AC, Cano Gutiérrez CA. Desigualdades entre mujeres y hombres mayores y menores de setenta años: Encuesta Salud, Bienestar y Envejecimiento (SABE) Colombia 2015. Univ Médica. 2019;60(3). https://doi.org/10.11144/J averiana.umed60-3.sabe

45. Castrillón-Torres GA, CadavidRamírez HJ. Proceso de paz entre gobierno colombiano y las FARC-EP: camino hacia la reincorporación de combatientes. Entramado. 2018;14(2):148-65.

46. Krug EG, Dahlberg LL, Mercy JA, Zwi AB, Lozano R. Informe mundial sobre la violencia y la salud. Rev Inst Med Trop Sao Paulo. 2003;45(3):130-130. 
47. Arango LM, Corrales Escobar L. Metacognición en el adulto mayor. Poiésis. 2014;1(28).

48. Blanco M. El enfoque del curso de vida: orígenes y desarrollo. Rev Latinoam Población. 2011;5(8):5-31.

49. Gutiérrez WA. Situación actual del médico geriatra en Colombia. Univ Med [internet]. 2015;56(2):163-77. Disponible en: https://revistas.javerian a.edu.co/index.php/vnimedica/article/ view/16355

50. Tatum PE, Talebreza S, Ross JS. Geriatric assessment: An officebased approach. Am Fam Physician. 2018;97(12).

51. Cervantes Becerra RG, Villarreal Ríos E, Galicia Rodríguez L, Vargas Daza ER, Martínez González L. Estado de salud en el adulto mayor en atención primaria a partir de una valoración geriátrica integral. Aten Primaria. 2015;47(6):329-35. http://dx .doi.org/10.1016/j.aprim.2014.07.007

52. Curcio CL, Pineda I A, María Rojas Z Á, Muñoz SL, Gómez F, Sanitas RM. Envejecer bien: un estudio sobre envejecimiento exitoso y sus determinantes. Rev Medica Sanitas. 2017;20(4):190-204.

53. Bastidas-Bilbao H. Enfermedades médicas y depresión en el adulto mayor: características comunes y relación etiológica. Revi Psicol. 2014;32 (2):191-218. https://doi.org/10 $.18800 /$ psico.201402.001

54. Brockie TN, Elm JHL, Walls ML. Examining protective and buffering associations between sociocultural factors and adverse childhood experiences among American Indian adults with type 2 diabetes: a quantitative, communitybased participatory research approach. BMJ Open. 2018
Sep;8(9):e022265. https://doi.org/10.1 136/bmjopen-2018-022265

55. McEniry M, Samper-Ternent R, Cano-Gutiérrez C. Displacement due to armed conflict and violence in childhood and adulthood and its effects on older adult health: The case of the middle-income country of Colombia. SSM Popul Health. 2019 Apr 1;7 (January). https://doi.org/10.10 16/j.ssmph.2019.100369 